\title{
Effects of Early Initiation of Solid Versus Liquid Diet after Endoscopic Submucosal Dissection on Quality of Life and Postoperative Outcomes: A Prospective Pilot Randomized Controlled Trial
}

\author{
Akihiro Miyakawa $^{a}$ Satoshi Kodera ${ }^{b, c}$ Yukie Sakuma ${ }^{b}$ Taro Shimada $^{a}$ \\ Manabu Kubota ${ }^{a}$ Akira Nakamura $^{a}$ Ei Itobayashi ${ }^{a}$ Haruhisa Shimura $^{a}$ \\ Yoshio Suzuki $^{d}$ Yasunori Sato $^{\text {e Kenji Shimura }}{ }^{a}$ \\ a Department of Gastroenterology, Asahi General Hospital, Chiba, Japan; ${ }^{b}$ Clinical Research Center, Asahi General \\ Hospital, Chiba, Japan; ' ${ }^{\circ}$ epartment of Cardiovascular Medicine, Graduate School of Medicine, The University of

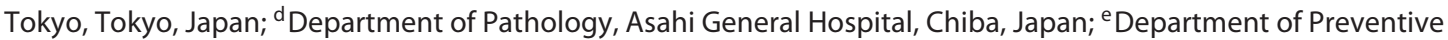 \\ Medicine and Public Health, Keio University School of Medicine, Tokyo, Japan
}

\section{Keywords}

Endoscopic submucosal dissection - Early solid diet .

Quality of life - Gastric neoplasms - Enhanced recovery after surgery

\begin{abstract}
Background/Aims: Feeding recommendations after endoscopic submucosal dissection (ESD) for gastric neoplasms are not established and based on clinical experience. Methods: This was a prospective pilot randomized controlled trial. Patients undergoing ESD for gastric neoplasms were randomly assigned to solid $(n=50)$ or liquid diet $(n=50)$ groups. Beginning the day after hemostasis confirmation until discharge, the solid diet group started on a diet of rice porridge, whereas the liquid diet group started on a liquid diet, with gradual transition to solid food. The primary endpoint was delayed bleeding rate. The secondary endpoints were quality of life (QOL), ulcer-stage, hospital fees, and post-ESD symptoms. Results: Delayed bleeding occurred in the solid diet group (2\%) but not in the liquid diet group. The QOL evaluation using European Organization for Research and Treatment of Cancer QLQ-C30 and QLQ-STO22 showed bet-
\end{abstract}

ter score in the solid diet group. The patients who felt dietary restriction at discharge was of a larger number in the liquid diet group ( $p=0.019)$. More patients experienced appetite loss $(p=0.038)$, constipation $(p=0.022)$, and dietary restriction $(p=0.037)$ in the liquid diet group during hospitalization. The other endpoints were equivalent between the groups. Conclusion: Early initiation of solid foods after ESD is feasible and associated with higher $\mathrm{QOL}$, potentially rendering conventional liquid diets unnecessary, although additional studies are needed (Trial registration number: UMIN000013297).

(c) 2018 The Author(s)
Published by S. Karger AG, Basel

\section{Introduction}

Currently, endoscopic resection is a widely accepted treatment for gastric neoplasms as it has a low-to-negligible risk of lymph node metastasis [1]. Endoscopic submucosal dissection (ESD) is widely used as an en bloc resection even in patients with large or ulcerative lesions, thereby reducing local recurrence [2-6]. Conventionally in Japan, to prevent delayed bleeding and promote post-

\begin{tabular}{|c|c|}
\hline KARGER & $\begin{array}{l}\text { (c) } 2018 \text { The Author(s) } \\
\text { Published by S. Karger AG, Basel }\end{array}$ \\
\hline $\begin{array}{l}\text { E-Mail karger@karger.com } \\
\text { www.karger.com/dig }\end{array}$ & $\begin{array}{l}\text { This article is licensed under the Creative Commons Attribution- } \\
\text { NonCommercial-NoDerivatives } 4.0 \text { International License (CC BY- } \\
\text { NC-ND) (http://www.karger.com/Services/OpenAccessLicense). } \\
\text { Usage and distribution for commercial purposes as well as any dis- } \\
\text { tribution of modified material requires written permission. }\end{array}$ \\
\hline
\end{tabular}

Akihiro Miyakawa

Department of Gastroenterology Asahi General Hospital

1326 I, Asahi, Chiba 289-2511 (Japan)

E-Mail a292miyaka2007@yahoo.co.jp 
ESD ulcer healing, patients undergoing ESD are instructed to fast afterwards, then start a liquid diet with gradual introduction of progressively more solid foods $[3,4]$. In Western countries, feeding recommendations for patients after ESD are generally based on Japanese clinical experience [5].

Although the early postoperative initiation of oral feeding is reported to decrease complications and provide higher quality of life (QOL) in the surgical field $[7,8]$, there are few standardized guidelines or studies on the optimal time to start oral intake after ESD. In addition, the necessity of gradually transitioning to solid foods after ESD has not been investigated to date.

Kim et al. [9] and Oh et al. [10] compared early feeding and fasting after ESD and showed that early feeding was associated with higher QOL and did not affect complication rates. However, they did not verify the necessity of gradually transitioning to increasingly solid foods, as was the focus of our study. Furthermore, they did not use objective indicators to evaluate QOL.

Early initiation of solid diet, by making high caloric intake possible and promoting gastric peristalsis, could possibly improve QOL. On the other hand, early initiation of solid diet might induce dyspepsia and increase adverse events, leading to reduce QOL. As a result, the advantages and disadvantages of early initiation of solid diet remain controversial. The feasibility of early initiation of solid diet might lead to decreased hospitalization durations and hospital fees. Therefore, the safety and effectiveness of early initiation of solid diet need to be validated.

This study sought to investigate the safety, effectiveness, objective QOL evaluation, and feasibility associated with early initiation of solid diet, compared with initiation of a liquid diet after ESD for gastric neoplasms.

\section{Materials and Methods}

\section{Patients}

Patients diagnosed with early-stage gastric cancer or adenoma by biopsy specimen who underwent ESD at Asahi General Hospital between March 2014 and August 2015 were enrolled in this study. All patients had normal organ function and an Eastern Cooperative Oncology Group performance status of 0 or 1 . This prospective pilot randomized controlled trial (RCT) was approved by the Institutional Review Board of Asahi General Hospital (November 20, 2013). Written informed consent for study participation was obtained from all patients prior to study procedures.

In our hospital, ESD was basically indicated for patients with gastric adenoma that was either $\geq 20 \mathrm{~mm}$ in size or depressed type; or early gastric cancer which met standard or expanded indication criteria according to Japanese gastric cancer treatment guidelines 2014 [11].

Early Solid and Liquid Diet on QOL and Post-ESD Outcomes
We enrolled consecutive patients with gastric adenoma or early gastric cancer who were treated with ESD and did not meet following exclusion criteria. The exclusion criteria were as follows: (I) use of antiplatelet agents, such as aspirin, thienopyridine derivatives, cilostazol, or anticoagulants; (II) pregnancy or nursing; (III) comorbid psychiatric disorder; (IV) oral administration of steroid drugs; and (V) active systemic infection. This prospective pilot RCT was registered with the University Hospital Medical Information Network Center in Japan (UMIN000013297; https://upload. umin.ac.jp/ctr).

\section{ESD Procedure}

An argon plasma coagulation probe was used for placement of the marking dots along the circumference of target lesion to indicate the margins. A mixed solution of hyaluronic acid sodium $(0.2 \%)$ containing $0.5 \%$ indigo carmine and $0.5 \%$ epinephrine was injected into the submucosal space to lift the lesion. This was followed by mucosal incision outside the marked margins. ESD was performed using an ITknife2 (KD-611L; Olympus Medical Systems, Tokyo, Japan), a DualKnife (KD-650L; Olympus), or an SB knife Jr (MD-47703W; Sumitomo Bakelite, Tokyo, Japan). A VIO300D electrosurgical generator (Erbe Elektromedizin, Tübingen, Germany) was used. The submucosal connective tissue was dissected, and the raised lesion was completely removed from the muscle layer.

\section{Perioperative Management}

Patients were admitted 1 day prior to ESD and remained in the hospital for 4 days after resuming oral intake. All patients received $20 \mathrm{mg}$ intravenous omeprazole for the first 2 days, beginning on the day of the procedure. Thereafter, $20 \mathrm{mg}$ rabeprazole per day was prescribed for 30 days.

\section{Study Design and Randomization}

The day after ESD, endoscopy was performed in all patients to evaluate the ulcer surface based on the modified Forrest classification, which has predictive value for peptic ulcer rebleeding (Fig. 1) [12]. This approach differentiates ulcers with spurting arterial hemorrhage (Forrest Ia) from those with oozing hemorrhage (Forrest Ib), a visible vessel (Forrest IIa), adherent clot (Forrest IIb), hematin on the ulcer base (Forrest IIc), or clean ulcer base (Forrest III). Hemostasis was performed for patients with ulcer surfaces rated Forrest Ia and Ib, and endoscopy was performed the next day for ulcer reevaluation. For patients rated Forrest IIa-III, hemostasis was performed as needed, and the patient resumed on oral intake the next day. For hemostasis, an EZ Clip (HX-610-135S; Olympus) or a Coagrasper (FD-411QR; Olympus) was used.

After confirmation of hemostasis, patients were randomized, on a 1:1 basis, to either the solid or the liquid diet using the envelope method. Patients in the solid diet group were started on rice porridge and solid side dishes the day after hemostasis confirmation until discharge. In contrast, patients in the liquid diet control group were started on a liquid diet the day after hemostasis confirmation and were gradually introduced to increasingly solid foods until discharge. Dietary protocols, components, and calories are shown in Table 1. All patients received dietary education at discharge to refrain from ingesting spicy and oily food within 4 weeks.

QOL was assessed using the Japanese version of the QLQ-C30 and QLQ-STO22 questionnaires related to gastric cancer, with 


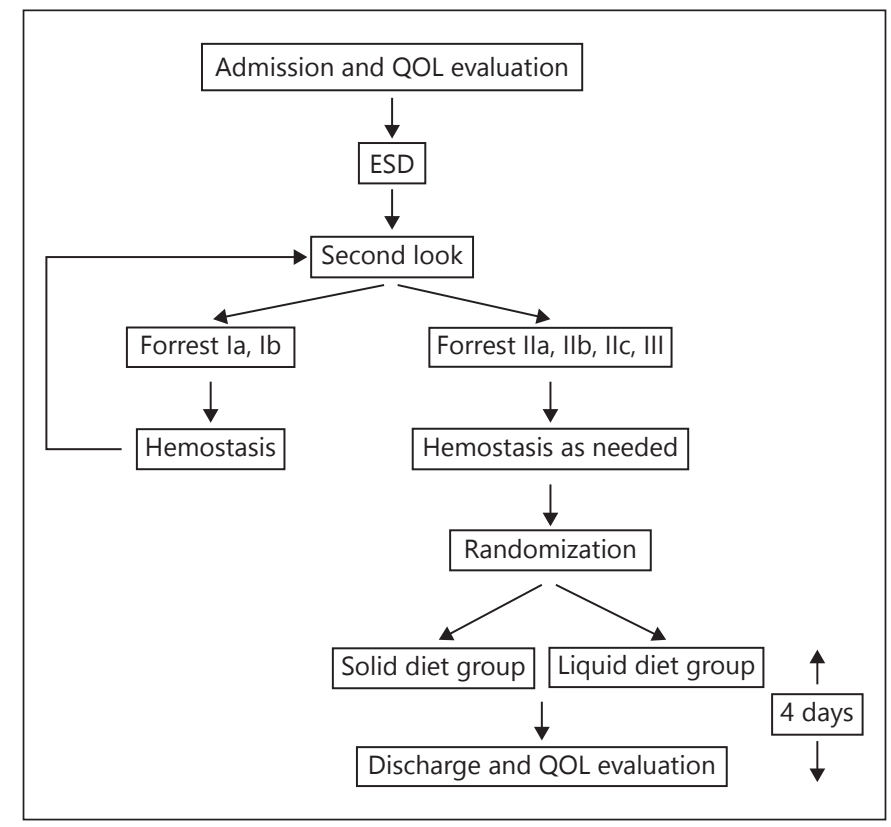

Fig. 1. Study design. The day after ESD, endoscopy was performed in all patients to evaluate the ulcer surface based on the modified Forrest classification, which has predictive value for peptic ulcer rebleeding. This approach differentiates ulcers with spurting arterial hemorrhage (Forrest Ia) from those with oozing hemorrhage (Forrest Ib), a visible vessel (Forrest IIa), adherent clot (Forrest IIb), hematin on the ulcer base (Forrest IIc), or clean ulcer base (Forrest III). Hemostasis was performed for patients with ulcer surfaces rated Forrest Ia and Ib, and endoscopy was performed the next day for ulcer reevaluation. For patients rated Forrest IIa-III, hemostasis was performed as needed, and the patient resumed on oral intake the next day. After confirmation of hemostasis, patients were randomized, on a 1:1 basis, to either the solid or the liquid diet using the envelope method. Patients in the solid diet group were started on rice porridge and solid side dishes the day after hemostasis confirmation until discharge. In contrast, patients in the liquid diet control group were started on a liquid diet the day after hemostasis confirmation and were gradually introduced to increasingly solid foods until discharge. QOL, quality of life; ESD, endoscopic submucosal dissection.

permission from the European Organization for Research and Treatment of Cancer (EORTC), the original designer of these instruments $[13,14]$. These questionnaires are frequently used for assessment QOL outcomes after ESD [15]. Patients answered these questionnaires at both admission and discharge. High scores on the functioning and global health status scales, as well as lower scores on the symptom scales, represent better QOL. For the EORTC QLQ-STO22, like the symptom scales, a high score represents low QOL. An additional questionnaire was given at discharge to evaluate diet satisfaction. The questionnaire assessed (I) hardness score between 1 and 3 ( 1 = hard, $2=$ neither hard nor soft, $3=$ soft) and (II) satisfaction score between 1 and 3 ( 1 = satisfied, $2=$ neither satisfied nor dissatisfied, 3 = dissatisfied). Endoscopy was performed approximately 4 weeks after ESD to assess ulcer stage.

\section{Endpoint and Outcome Evaluation}

The primary endpoint was delayed bleeding rate. The secondary endpoints were change in QOL between the date of admission and hospital discharge, ulcer stage at 4 weeks after ESD as per Sakita-Fukutomi classification, hospital fees, days of hospitalization, and post-ESD symptoms including abdominal distension, abdominal pain, constipation, diarrhea, and nausea. The delayed bleeding was defined as hematemesis or melena requiring endoscopic hemostasis or blood transfusion or leading to a reduction in hemoglobin by more than $2 \mathrm{~g} / \mathrm{dL}$. Sakita-Fukutomi classification is commonly used in East Asia during endoscopic evaluations to determine the likelihood of healing tendency for peptic ulcers. This classification differentiates among A1 (ulcer covered with thick slough and edematous border, sometimes with clot, visible vessel, or bleeding), A2 (ulcer covered with slough and less edematous than A1), H1 (ulcer covered with $<50 \%$ regenerating epithelium), $\mathrm{H} 2$ (ulcer with a mucous break but covered with $>50 \%$ regenerating epithelium), S1 (red scar), and S2 (white scar). We defined $\mathrm{A} 1-\mathrm{H} 1$ as the active stage and $\mathrm{H} 2-\mathrm{S} 2$ as the healing stage. The ulcer stage at 4 weeks after ESD was evaluated by 3 board-certified fellows of the Japan Gastroenterological Endoscopy Society who were blind to subject identity or group assignment. Post-ESD symptoms were evaluated using the CTCAE version 4.0.

\section{Statistical Analysis}

We hypothesized that a liquid diet was not necessary for patients with confirmed hemostasis after ESD. A liquid diet would be judged not necessary if the solid diet group exhibited superior results for the endpoints.

Statistical analysis and reporting of this study were conducted in accordance with the CONSORT guidelines. The sample size was based upon feasibility and rapid patient recruitment, considering the pilot study period within one and a half years. The patients undergoing ESD in our hospital per year are approximately 120 . Assuming that $30 \%$ of patients would not meet inclusion criteria, $10 \%$ would not agree study concept, and 5\% would be lost to follow-up, the sample size was determined to be 100 patients.

The analyses were conducted using Fisher's exact test, unpaired $t$ tests, paired $t$ tests and the chi-square test, as appropriate at the Clinical Research Support Center of Asahi General Hospital. All comparisons were planned, and all $p$ values were two sided. A $p$ value of $<0.05$ was considered statistically significant. All statistical analyses were performed using JMP ${ }^{\circledR}$ software version 10.0.2 (SAS Institute Japan, Tokyo, Japan).

\section{Results}

\section{Clinical Characteristics of the Patients in the Solid}

Diet Group and Liquid Diet Group

A flowchart describing patient inclusion and exclusion criteria used in this study is presented in Figure 2. One hundred ten patients were enrolled in the study. The age of patients ranged from 20 to 90 years. Three patients withdrew informed consent and 2 violated the inclusion criteria before ESD, leaving 105 patients treated with ESD. One patient sustained an injured muscle layer during ESD, 2 pa- 
Fig. 2. Flowchart. One hundred and ten patients were enrolled in the study. Three patients withdrew informed consent and 2 violated the inclusion criteria before ESD, leaving 105 patients treated with ESD. One patient sustained an injured muscle layer during ESD, 2 patients had uncompleted ESDs due to fibrosis, one did not complete ESD due to bleeding, and one had a deep post-ESD ulcer at second-look endoscopy. Almost all patients adhered to the study protocol after ESD. Only 1 patient with a Forrest Ib ulcer due to loss of visible vessels during second-look endoscopy did not achieve hemostasis; third-look endoscopy was performed as a part of the protocol. In total, 100 patients undergoing ESD were randomly assigned to either the solid diet $(n=50)$ or the liquid diet $(n=50)$ group. One patient underwent colorectal surgery after ESD in the liquid diet group, and thus 49 patients underwent endoscopy 4 weeks after ESD. Finally, 100 patients were included in the intention-to-treat analysis of the endpoints. ESD, endoscopic submucosal dissection.

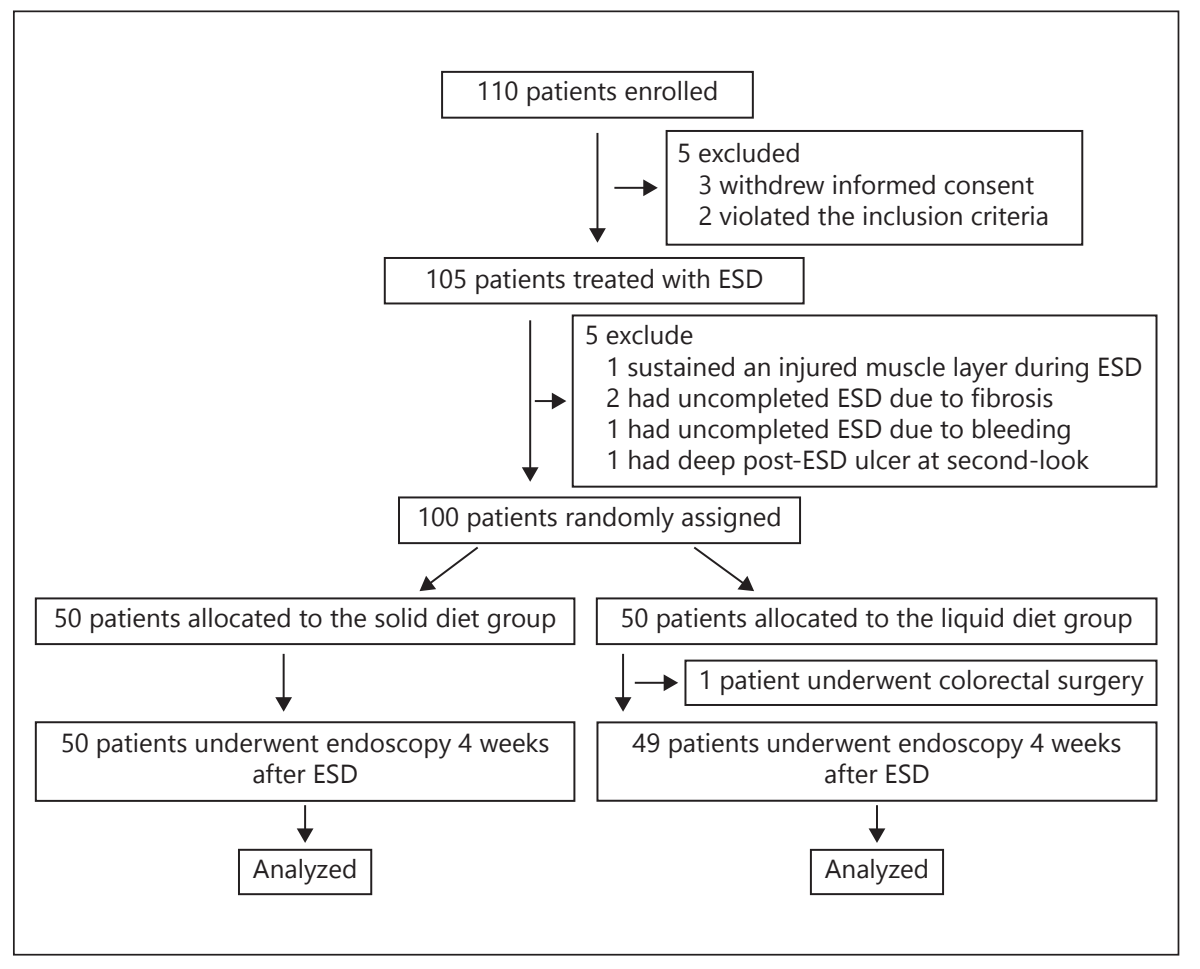

Table 1. Dietary protocols, components, and calories of foods

\begin{tabular}{llll}
\hline & Breakfast & Lunch & Dinner \\
\hline Solid diet group & & & \\
Day 1 & E & E & E \\
Day 2 & E & E & E \\
Day 3 & E & E & \\
Day 4 & E & & A \\
Liquid diet group & A & A & D \\
Day 1 & B & B & Total calories, kcal \\
Day 2 & C & Discharge & \\
Day 3 & E & Side dish, kcal & 36 \\
Day 4 & Staple diet, kcal & & 299 \\
& & 0 & 346 \\
\hline Calorie of staple diet and side dish & 36 & 266 & 367 \\
A & 33 & 292 & 398 \\
B & 54 & 292 & \\
C & 75 & 292 & \\
D & 106 & & \\
E & & & \\
\hline A: liquid diet. & & & \\
B: rice porridge in 30\% degree. & & & \\
C: rice porridge in half degree. & & & \\
D: rice porridge in 70\% degree. & & & \\
E: rice porridge. & & & \\
\hline
\end{tabular}


Table 2. Comparison of patient characteristics in the solid and liquid diet groups

\begin{tabular}{|c|c|c|c|}
\hline Category & Solid diet $(n=50)$ & Liquid diet $(n=50)$ & $p$ value \\
\hline Age, years & $72.4 \pm 9.0$ & $70.5 \pm 10.1$ & $0.304^{\dagger}$ \\
\hline \multicolumn{4}{|l|}{ Gender } \\
\hline Male & $36(72)$ & $31(62)$ & $0.395^{\ddagger}$ \\
\hline Female & $14(28)$ & $19(38)$ & \\
\hline Body mass index, $\mathrm{kg} / \mathrm{m}^{2}$ & $23.8 \pm 3.7$ & $24.0 \pm 3.6$ & $0.729^{\dagger}$ \\
\hline Serum albumin, $\mathrm{g} / \mathrm{dL}$ & $4.4 \pm 0.4$ & $4.3 \pm 0.4$ & $0.980^{\dagger}$ \\
\hline Prognostic nutritional index & $52.7 \pm 5.0$ & $52.2 \pm 5.2$ & $0.585^{\dagger}$ \\
\hline Drinking habit & $26(52)$ & $21(42)$ & $0.423^{\ddagger}$ \\
\hline Smoking & $8(16)$ & $7(14)$ & $>0.999 \ddagger$ \\
\hline \multicolumn{4}{|l|}{ Comorbidities } \\
\hline Hypertension & $30(60)$ & $30(60)$ & $>0.999^{\ddagger}$ \\
\hline Diabetes mellitus & $10(20)$ & $8(16)$ & $0.795^{\ddagger}$ \\
\hline Hyperlipidemia & $18(36)$ & $18(36)$ & $>0.999^{\ddagger}$ \\
\hline $\begin{array}{l}\text { Data are presented as mea } \\
{ }^{\dagger} \text { Unpaired } t \text { test. } \\
{ }^{\ddagger} \text { Fisher's exact test. }\end{array}$ & $r$ the number $(\%)$. & & \\
\hline
\end{tabular}

tients had uncompleted ESDs due to fibrosis, one did not complete ESD due to bleeding, and one had a deep postESD ulcer at second-look endoscopy. Almost all patients adhered to the study protocol after ESD. Only 1 patient with a Forrest Ib ulcer due to loss of visible vessels during secondlook endoscopy did not achieve hemostasis; third-look endoscopy was performed as a part of the protocol. In total, 100 patients undergoing ESD were randomly assigned to either the solid diet $(n=50)$ or the liquid $\operatorname{diet}(n=50)$ group. One patient underwent colorectal surgery after ESD in the liquid diet group; thus, 49 patients underwent endoscopy 4 weeks after ESD. Finally, 100 patients were included in the intention-to-treat analysis of the endpoints.

Baseline characteristics of the patients are presented in Table 2. Lesion characteristics and the results of the ESD procedure are summarized in Table 3. Patient characteristics and tumor- and treatment-related factors were well balanced between the groups. A total of 2 surgeons participated in this study, and they operated on an approximately equal number of patients from each group. The amount of dietary intake was statistically equivalent between the groups.

\section{Assessment of QOL}

EORTC scores are shown in Table 4. The number of patients who felt they were going through a dietary restriction at discharge was higher in the liquid diet group than in the solid diet group $(p=0.019)$. Patients on the liquid diet experienced significantly greater appetite loss $(p=0.038)$, constipation $(p=0.022)$, and dietary restric- tion ( $p=0.037)$ than patients on the solid diet group during hospitalization. The symptoms of dyspepsia including nausea, vomiting, pain, and reflux symptoms were statistically equivalent between the groups. In the questionnaires to evaluate diet satisfaction at discharge, the number of patients who reported their diets were too soft was significantly higher in the liquid diet group than in the solid diet group ( 46 vs. $14 \%, p<0.001$ ). The solid diet group had higher satisfaction than the liquid diet group (88 vs. $40 \%, p<0.001$ ).

\section{Adverse Events}

Delayed bleeding occurred in 1 patient $(2 \%)$ in the solid diet group (95\% CI $0.4-10.5 \%$ ) but did not occur the liquid diet group. The patient with delayed bleeding was hospitalized due to tarry stool on postoperative day 9 and hematemesis on postoperative day 10. Emergency endoscopy did not reveal visible vessels within the ulcer, and hemostasis was unnecessary. Post-ESD stenosis occurred in 1 patient in each group (2\%). Both patients required frequent endoscopic balloon dilation, and symptoms of obstruction disappeared after treatment. Other adverse events were not significantly different between the groups, as summarized in Table 5. Hospital fees was statistically equivalent between the groups.

\section{Post-ESD Ulcer in the Solid Diet Group and Liquid Diet Group}

Although there were no significant differences in the healing rate of post-ESD ulcer between the 2 groups, there 
Table 3. Comparison of oncological factors and surgical factors in the solid and liquid diet groups

\begin{tabular}{|c|c|c|c|}
\hline Category & Solid diet $(n=50)$ & Liquid diet $(n=50)$ & $p$ value \\
\hline Specimen size, $\mathrm{mm}$ & $42.4 \pm 15.6$ & $41.9 \pm 13.4$ & $0.864^{\dagger}$ \\
\hline Tumor size, mm & $17.7 \pm 10.9$ & $17.0 \pm 10.6$ & $0.735^{\dagger}$ \\
\hline Gross type $(0-\mathrm{I} / 0-\mathrm{IIa} / 0-\mathrm{IIb} / 0-\mathrm{II} c / 0-\mathrm{IIa}+\mathrm{IIc})$ & $2 / 14 / 3 / 29 / 2$ & $2 / 6 / 2 / 33 / 7$ & $0.162^{\ddagger}$ \\
\hline Location (upper/middle/lower) & $7 / 15 / 28$ & $6 / 12 / 32$ & $0.713^{\S}$ \\
\hline Pathology (adenocarcinoma/adenoma/other) & $39 / 8 / 3$ & $40 / 9 / 1$ & $0.726^{\ddagger}$ \\
\hline Depth (M/SM1/SM2) & $34 / 2 / 3$ & $33 / 3 / 4$ & $>0.999^{\ddagger}$ \\
\hline Pathological ulceration & $17(34)$ & $18(36)$ & $>0.999^{\ddagger}$ \\
\hline Lymphatic invasion & $1(2)$ & $3(6)$ & $0.615^{\ddagger}$ \\
\hline Vascular invasion & $1(2)$ & $3(6)$ & $0.615^{\ddagger}$ \\
\hline Curability (complete/expanded/incomplete/other) & $23 / 16 / 8 / 3$ & $22 / 20 / 7 / 1$ & $0.715^{\ddagger}$ \\
\hline Days of hospitalization after ESD & $5.2 \pm 0.5$ & $5.3 \pm 0.7$ & $0.246^{\dagger}$ \\
\hline Ulcer stage at 4 weeks after ESD (active/healing) & $35 / 15$ & $42 / 7$ & $0.089^{\ddagger}$ \\
\hline Hospital fees (Japanese Yen) & $460,141 \pm 77,185$ & $467,998 \pm 77,194$ & $0.612^{\dagger}$ \\
\hline Hemorrhage at second look (Forrest Ia/Ib/IIa/IIb/IIc/III) & $2 / 6 / 14 / 25 / 0 / 3$ & $0 / 12 / 10 / 26 / 0 / 2$ & $0.311^{\ddagger}$ \\
\hline Hemostasis by endoscopy (prevention for Forrest IIa-III) & $14(28)$ & $9(18)$ & $0.342^{\ddagger}$ \\
\hline Hemostasis by endoscopy (treat for Forrest Ia-Ib) & $8(16)$ & $11(22)$ & $0.611^{\ddagger}$ \\
\hline Intake of staple diet, $\%$ & $94.2 \pm 11.1$ & $94.4 \pm 10.9$ & $0.930^{\dagger}$ \\
\hline Intake of side dish, $\%$ & $91.8 \pm 12.5$ & $90.7 \pm 12.9$ & $0.690^{\dagger}$ \\
\hline $\begin{array}{l}\text { Data are presented as mean } \pm \text { SD or the number }(\%) \text {. } \\
\text { 0-I, protruding; } 0 \text {-IIa, superficial elevated; } 0-\mathrm{IIb} \text {, super } \\
\text { M, intramucosal; SM1, <500 micron from the muscula } \\
\text { mucosae; ESD, endoscopic submucosal dissection. } \\
{ }^{\dagger} \text { Unpaired } t \text { test. } \\
{ }^{\ddagger} \text { Fisher's exact test. } \\
{ }^{\S} \text { Chi-square test. }\end{array}$ & $\begin{array}{l}\text { ficial flat; } 0-I I c, \text { supe } \\
\text { is mucosae; SM2, } \geq 5\end{array}$ & $\begin{array}{l}\text { rficial depressed. } \\
00 \text { micron from the } \mathrm{n}\end{array}$ & nuscularis \\
\hline
\end{tabular}

was a trend in healing improvement in the solid diet group compared with that in the liquid diet group, as presented in Table $3(p=0.089)$.

\section{Discussion}

To the best of our knowledge, this is the first prospective pilot RCT to investigate the difference in adverse event frequency and QOL changes after ESD between patients on solid and liquid diets. Our results showed that early initiation of a solid diet was feasible and associated with better QOL compared with conventional clinical pathway, which involved a liquid diet, followed by the gradual introduction of solid foods.

In the surgical field, the importance of enhanced recovery after surgery (ERAS) has been argued for a long time [16]. It is reported that ERAS contributed to a decrease in postoperative complications and improved QOL. Therefore, early postoperative initiation of oral feeding is recommended in the surgical field as ERAS programs.

Early Solid and Liquid Diet on QOL and Post-ESD Outcomes
The American Society of Colon and Rectal Surgeons and Society of American Gastrointestinal and Endoscopic Surgeons based on the several studies strongly recommends that following a regular diet immediately after colorectal surgery showed that early feeding after colorectal surgery was useful, safe, and well-tolerated [17]. While the evidence for early oral regular diet after colorectal surgery has been established, its application for gastric surgery was limited because of a fear of possible leakage due to mechanical stimulation and incrementally increased intraluminal pressure. However, a number of recent studies reported the feasibility and efficacy of early oral feeding after gastric surgery. Experimental and clinical data in rats suggested that early postoperative oral feeding accelerates upper gastrointestinal anastomotic healing [18]. Several studies, including a meta-analysis have shown that early postoperative initiation of oral feeding is associated with lower complication rates and higher QOL [7, 8, 19-22]. However, the necessity of gradually transitioning to increasingly solid foods, as was the focus of this study, has not been investigated to date. 
Table 4. European organization for research and treatment of cancer score in the solid and liquid diet groups

\begin{tabular}{|c|c|c|c|c|c|c|}
\hline \multirow[t]{2}{*}{ Category } & \multicolumn{3}{|c|}{$\begin{array}{l}\text { Score changing between admission and dischar- } \\
\text { ge }\end{array}$} & \multicolumn{3}{|l|}{ At discharge } \\
\hline & $\begin{array}{l}\text { solid diet } \\
(n=50)\end{array}$ & $\begin{array}{l}\text { liquid diet } \\
(n=50)\end{array}$ & $\begin{array}{l}p \text { values } \\
\text { (paired-t) }\end{array}$ & $\begin{array}{l}\text { solid diet } \\
(n=50)\end{array}$ & $\begin{array}{l}\text { liquid diet } \\
(n=50)\end{array}$ & $\begin{array}{l}p \text { value } \\
\text { (unpaired-t) }\end{array}$ \\
\hline \multicolumn{7}{|l|}{ EORTC QLQ-C30 } \\
\hline Global health status & $1.50 \pm 20.53$ & $-5.00 \pm 20.96$ & 0.120 & $58.50 \pm 16.37$ & $57.17 \pm 17.17$ & 0.692 \\
\hline \multicolumn{7}{|l|}{ Functional scales } \\
\hline Physical functioning & $-0.13 \pm 8.36$ & $-0.53 \pm 8.39$ & 0.812 & $96.00 \pm 8.19$ & $95.33 \pm 8.44$ & 0.689 \\
\hline Role functioning & $-1.00 \pm 4.00$ & $-2.00 \pm 10.98$ & 0.547 & $98.67 \pm 4.57$ & $96.33 \pm 10.80$ & 0.163 \\
\hline Emotional functioning & $2.33 \pm 10.52$ & $2.50 \pm 11.07$ & 0.939 & $90.50 \pm 11.17$ & $92.50 \pm 9.26$ & 0.332 \\
\hline Cognitive functioning & $2.33 \pm 11.17$ & $1.33 \pm 4.57$ & 0.559 & $98.00 \pm 8.00$ & $97.67 \pm 8.92$ & 0.844 \\
\hline Social functioning & $2.00 \pm 13.74$ & $1.33 \pm 12.07$ & 0.797 & $92.00 \pm 11.29$ & $92.33 \pm 13.56$ & 0.894 \\
\hline \multicolumn{7}{|l|}{ Symptom scales } \\
\hline Fatigue & $0.44 \pm 10.28$ & $-0.22 \pm 9.39$ & 0.736 & $4.89 \pm 8.14$ & $5.11 \pm 7.52$ & 0.888 \\
\hline Nausea and vomiting & $2.00 \pm 9.31$ & $2.00 \pm 10.45$ & $>0.999$ & $2.67 \pm 8.49$ & $2.00 \pm 10.45$ & 0.727 \\
\hline Pain & $0.00 \pm 14.29$ & $1.00 \pm 17.95$ & 0.759 & $7.67 \pm 13.97$ & $6.33 \pm 11.11$ & 0.599 \\
\hline Dyspnea & $0.67 \pm 8.22$ & $-2.00 \pm 12.44$ & 0.209 & $1.33 \pm 6.60$ & $2.67 \pm 11.35$ & 0.474 \\
\hline Insomnia & $6.67 \pm 22.34$ & $10.00 \pm 27.15$ & 0.504 & $15.33 \pm 24.48$ & $18.00 \pm 26.26$ & 0.601 \\
\hline Appetite loss & $-2.00 \pm 14.14$ & $4.00 \pm 14.51$ & 0.039 & $1.33 \pm 6.60$ & $5.33 \pm 14.06$ & 0.072 \\
\hline Constipation & $3.33 \pm 27.97$ & $16.67 \pm 29.55$ & 0.023 & $19.33 \pm 24.36$ & $25.33 \pm 29.01$ & 0.266 \\
\hline Diarrhea & $2.67 \pm 20.02$ & $4.00 \pm 22.98$ & 0.758 & $7.33 \pm 15.49$ & $8.67 \pm 18.82$ & 0.700 \\
\hline Financial difficulties & $-4.67 \pm 15.07$ & $0.00 \pm 17.82$ & 0.161 & $2.00 \pm 8.00$ & $4.67 \pm 20.21$ & 0.388 \\
\hline \multicolumn{7}{|l|}{ EORTC QLQ-STO22 } \\
\hline \multicolumn{7}{|l|}{ Symptom scales } \\
\hline Dysphagia & $-0.22 \pm 2.74$ & $0.67 \pm 3.48$ & 0.159 & $0.22 \pm 1.57$ & $1.11 \pm 3.37$ & 0.094 \\
\hline Pain & $1.33 \pm 11.83$ & $4.50 \pm 12.17$ & 0.190 & $8.00 \pm 11.03$ & $9.33 \pm 11.86$ & 0.562 \\
\hline Reflux & $-1.11 \pm 7.19$ & $-1.11 \pm 5.14$ & $>0.999$ & $1.56 \pm 4.50$ & $1.33 \pm 3.65$ & 0.787 \\
\hline Eating & $-0.50 \pm 5.93$ & $3.33 \pm 11.42$ & 0.038 & $2.00 \pm 4.63$ & $5.50 \pm 9.31$ & 0.019 \\
\hline Anxiety & $0.22 \pm 15.63$ & $2.00 \pm 18.87$ & 0.609 & $22.00 \pm 17.75$ & $23.33 \pm 17.85$ & 0.709 \\
\hline Dry mouth & $-2.67 \pm 17.61$ & $-0.67 \pm 14.27$ & 0.534 & $10.00 \pm 16.84$ & $8.00 \pm 14.38$ & 0.524 \\
\hline Taste & $-1.33 \pm 6.60$ & $0.67 \pm 12.58$ & 0.322 & $0.67 \pm 4.71$ & $2.67 \pm 9.13$ & 0.172 \\
\hline Body image & $0.67 \pm 4.71$ & $-1.33 \pm 16.44$ & 0.410 & $0.67 \pm 4.71$ & $2.67 \pm 11.35$ & 0.253 \\
\hline Hair loss & $0.33 \pm 2.36$ & $0.33 \pm 7.13$ & $>0.999$ & $0.33 \pm 2.36$ & $1.67 \pm 8.42$ & 0.283 \\
\hline
\end{tabular}

Data are presented as mean $\pm \mathrm{SD}$.

QOL, quality of life; EORTC, european organization for research and treatment of cancer.

Although endoscopists strongly promote ESD because of higher QOL, few RCTs for oral intake after endoscopic procedures leading to QOL improvement exist. To the best of our knowledge, only 2 RCTs verified oral intake after ESD. Kim et al. [9] and Oh et al. [10] compared early feeding and fasting after ESD and showed that early feeding was associated with higher QOL, was more cost effective, decreased hospital stays, and did not influence complication rates. However, there are no studies about the efficacy and safety of early intake of solid foods. Therefore, establishing evidence for oral intake after ESD, similar to that of gastric surgery, is highly anticipated.

Body weight loss after gastric surgery seems to relate to lower QOL and reduced 5-year survival rates [23, 24].
A nutritious diet is necessary after gastric surgery for the prevention of body weight loss. Imamura et al. [25] reported that $300 \mathrm{kcal}$ daily elemental diet supplementation reduced postoperative weight loss in gastric cancer patients undergoing gastrectomy. Adachi et al. [26] suggested that administration of synthetic ghrelin improves appetite and increases calorie intake, thereby lessening body weight loss. We conducted this study for the purpose of achieving adequate nutrition after ESD. The total calories consumed per day were significantly higher in the solid diet group than in the liquid diet group (first day: 1,194 vs. $108 \mathrm{kcal} /$ day, total: 3,980 vs. $2,530 \mathrm{kcal}$ ). The report that early resumption of oral intake helps reduce postoperative weight loss and complication rates might be asso- 
Table 5. Adverse events in the solid and liquid diet groups

\begin{tabular}{llcc}
\hline Category & Solid diet $(n=50)$ & Liquid diet $(n=50)$ & $p$ value (Fisher's exact test) \\
\hline Adverse event after ESD & & & \\
Delayed bleeding (grade 1) & $1(2)^{*}$ & $0(0)$ & $>0.999$ \\
Blood transfusion & $0(0)$ & $0(0)$ & $>0.999$ \\
Hemostasis by IVR & $0(0)$ & $0(0)$ & $>0.999$ \\
Hemostasis by operation & $0(0)$ & $0(0)$ & $>0.999$ \\
Stenosis (grade 3) & $1(2)$ & $1(2)$ & $>0.999$ \\
Perforation & $0(0)$ & $0(0)$ & $>0.999$ \\
Adverse event after oral intake & & $9(18)$ & 0.388 \\
Nausea (grade 1) & $5(10)$ & $5(10)$ & $>0.999$ \\
Abdominal pain (grade 1) & $4(8)$ & $0(24)$ & $>0.999$ \\
Constipation (grade 1) & $6(12)$ & $1(2)$ & 0.499 \\
Diarrhea & $0(0)$ & $0(0)$ & \\
Fever (grade 1) & $2(4)$ & & \\
Fever (grade 2) & $2(4)$ & & \\
\hline &
\end{tabular}

ciated with the total calories intake after gastric surgery. Early resumption of solid foods after ESD may be a nutritious and effective strategy, if safety can be assured.

A novel finding of this study was the improved QOL in the solid diet group. This is the first RCT to verify a higher QOL in the solid diet group using objective indicators. A nutritious and satisfying solid diet might improve appetite and promote bowel peristalsis, leading to decreased constipation.

Although there were no significant differences, there was a trend toward improved post-ESD ulcer healing in the solid diet group. Many fear that early resumption of solid foods might directly stimulate the ulcer and induce bleeding while also promoting gastric peristalsis to induce wound opening, potentially culminating in delayed wound healing. On the other hand, proper nutrition might improve healing and solid foods within the stomach may neutralize gastric acids and cover the ulcer. Thus, the relationship between early resumption of solid foods and wound healing remains controversial. Kim et al. [9] and Oh et al. [10] reported that the post-ESD ulcer 2 months after surgery was statistically equivalent between the early feeding and fasting groups. This is the first study to demonstrate that early resumption of solid foods after ESD may improve ulcer healing. This outcome should be validated in a multicenter RCT that includes multimodal care.

The delayed bleeding rate in the solid diet group (2\%; 95\% CI 0.4-10.5\%) was considered clinically acceptable considering previously reported rates of $0-15.6 \%[2-5,9$, $10,15,27-34]$. There was no significant difference between the solid and liquid diet groups in terms of the frequency of adverse events. The largest specimen size in the solid diet group was $100 \times 65 \mathrm{~mm}$. In addition, an early solid diet was well-tolerated, even in patients with postESD ulcers that were over $100 \mathrm{~mm}$ in size. These results suggest that an early solid diet might not stimulate the post-ESD ulcer directly, thereby not inducing bleeding.

Both groups were set to equal numbers of days of hospitalization after ESD. Our results found no early bleeding after resumption of solid foods; this finding (if further verified) might lead to decreased hospitalization durations and hospital fees in the future.

There were several limitations in this study. First, the definition of feasibility was ambiguous. Although the adverse events and dyspeptic symptoms revealed no significant difference between the groups, the hypothesis that routine liquid diet intake following ESD was unnecessary would require verification of non-inferiority of the solid diet group to the liquid diet group in adverse events. Second, the basic nutritional assessment used in this study is inadequate. The difference in albumin, prealbumin and body weight between admission and discharge should be assessed. Third, this study design included a second-look endoscopy. The second-look endoscopy after ESD has not been routinely recommended, since the results of the SAFE trial were reported [34]. However, the SAFE trial 
did not investigate the early intake of solid foods after ESD. In the absence of evidence associated with the safety and tolerability of early resumption of solid foods, this study design is considered to be acceptable. Fourth, this was a single-center study. Therefore, a multicenter RCT that includes multimodal care might provide additional clinical evidence supporting early resumption of solid foods after ESD.

In conclusion, we demonstrated that the early resumption of a solid diet after ESD is feasible. Consuming solid foods after ESD improves QOL and might accelerate wound healing in patients with gastric neoplasms. Conventional use of liquid diets might not be necessary if hemostasis can be confirmed after ESD. Further large-scale comparison studies are needed to confirm its efficacy.

\section{Acknowledgments}

We thank Dr. Tomonori Yano (Department of Gastroenterology and Endoscopy at the National Cancer Center Hospital East, Kashiwa, Japan) for his insightful comments.

This study was supported by the Clinical Research Support Center at Asahi General Hospital, Chiba, Japan. We thank all support team members.

\section{Disclosure Statement}

The authors report no conflict of interest. This is not a collaborative study. There is neither financial support nor interest from any companies. We have no conflicts of interest or financial ties to disclose.

\section{References}

1 Gotoda T, Yanagisawa A, Sasako M, Ono H, Nakanishi Y, Shimoda T, et al: Incidence of lymph node metastasis from early gastric cancer: estimation with a large number of cases at two large centers. Gastric Cancer 2000;3:219225 .

2 Oda I, Gotoda T, Hamanaka H, Eguchi T, Saito Y, Matsuda T, et al: Endoscopic submucosal dissection for early gastric cancer: technical feasibility, operation time and complications from a large consecutive series. Dig Endosc 2005; 17:54-58.

3 Fujishiro M: Endoscopic submucosal dissection for stomach neoplasms. World J Gastroenterol 2006; 12:5108-5112.

4 Gotoda T: Endoscopic resection of early gastric cancer. Gastric Cancer 2007;10:1-11.

5 Coda S, Lee SY, Gotoda T: Endoscopic mucosal resection and endoscopic submucosal dissection as treatments for early gastrointestinal cancers in Western countries. Gut Liver 2007; 1:12-21.

6 Tanaka M, Ono H, Hasuike N, Takizawa K: Endoscopic submucosal dissection of early gastric cancer. Digestion 2008;77:23-28.

7 Hur H, Kim SG, Shim JH, Song KY, Kim W, Park CH, et al: Effect of early oral feeding after gastric cancer surgery: a result of randomized clinical trial. Surgery 2011;149:561-568.

8 Kim JW, Kim WS, Cheong JH, Hyung WJ, Choi SH, Noh SH: Safety and efficacy of fasttrack surgery in laparoscopic distal gastrectomy for gastric cancer: a randomized clinical trial. World J Surg 2012;36:2879-2887.

9 Kim S, Cheoi KS, Lee HJ, Shim CN, Chung HS, Lee H, et al: Safety and patient satisfaction of early diet after endoscopic submucosal dissection for gastric epithelial neoplasia: a prospective, randomized study. Surg Endosc 2014;28:1321-1329.

10 Oh KH, Lee SJ, Park JK: Optimal duration of fasting period after endoscopic submucosal dissection for gastric epithelial neoplasia: A prospective evaluation. J Dig Dis 2017;18: 445-452.

11 Japanese Gastric Cancer Association: Japanese gastric cancer treatment guidelines 2014 (ver. 4). Gastric Cancer 2017;20:1-9.

12 de Groot NL, van Oijen MG, Kessels K, Hemmink M, Weusten BL, Timmer R, et al: Reassessment of the predictive value of the Forrest classification for peptic ulcer rebleeding and mortality: can classification be simplified? Endoscopy 2014;46:46-52.

13 Vickery CW, Blazeby JM, Conroy T, Arraras J, Sezer O, Koller M, et al: Development of an EORTC disease-specific quality of life module for use in patients with gastric cancer. Eur J Cancer 2001;37:966-971.

14 Blazeby JM, Conroy T, Bottomley A, Vickery C, Arraras J, Sezer O, et al: Clinical and psychometric validation of a questionnaire module, the EORTC QLQ-STO 22, to assess quality of life in patients with gastric cancer. Eur J Cancer 2004;40:2260-2268.

15 Kim SG, Ji SM, Lee NR, Park SH, You JH, Choi IJ, et al: Quality of ife after endoscopic submucosal dissection for early gastric cancer: a prospective multicenter cohort study. Gut Liver 2017;11:87-92.

16 Ljungqvist O, Scott M, Fearon KC: Enhanced recovery after surgery: a review. JAMA Surg 2017;152:292-298.

17 Carmichael JC, Keller DS, Baldini G, Bordeianou L, Weiss E, Lee L, et al: Clinical practice guideline for enhanced recovery after colon and rectal surgery from the American Society of Colon and Rectal Surgeons (ASCRS) and Society of American Gastrointestinal and Endoscopic Surgeons (SAGES). Surg Endosc 2017;31:3412-3436.

18 Fukuzawa J, Terashima H, Ohkohchi N: Early postoperative oral feeding accelerates upper gastrointestinal anastomotic healing in the rat model. World J Surg 2007;31:12341239.

19 Liu X, Wang D, Zheng L, Mou T, Liu H, Li G: Is early oral feeding after gastric cancer surgery feasible? A systematic review and metaanalysis of randomized controlled trials. PLoS One 2014;9:e112062.

20 Chen ZX, Liu AH, Cen Y: Fast-track program vs traditional care in surgery for gastric cancer. World J Gastroenterol 2014;20:578-583.

21 Ding J, Sun B, Song P, Liu S, Chen H, Feng M, et al: The application of enhanced recovery after surgery (ERAS)/fast-track surgery in gastrectomy for gastric cancer: a systematic review and meta-analysis. Oncotarget 2017;8: 75699-75711.

22 Li Z, Wang Q, Li B, Bai B, Zhao Q: Influence of enhanced recovery after surgery programs on laparoscopy-assisted gastrectomy for gastric cancer: a systematic review and metaanalysis of randomized control trials. World J Surg Oncol 2017;15:207.

23 Kong H, Kwon OK, Yu W: Changes of quality of life after gastric cancer surgery. J Gastric Cancer 2012;12:194-200.

$24 \mathrm{Yu} \mathrm{W}$, Seo BY, Chung HY: Postoperative body-weight loss and survival after curative resection for gastric cancer. Br J Surg 2002;89: 467-470.

25 Imamura H, Nishikawa K, Kishi K, Inoue K, Matsuyama J, Akamaru Y, et al: Effects of an oral elemental nutritional supplement on post-gastrectomy body weight loss in gastric cancer patients: a randomized controlled clinical trial. Ann Surg Oncol 2016;23:29282935.

26 Adachi S, Takiguchi S, Okada K, Yamamoto $\mathrm{K}$, Yamasaki M, Miyata $\mathrm{H}$, et al: Effects of ghrelin administration after total gastrectomy: a prospective, randomized, placebo-controlled phase II study. Gastroenterology 2010; 138:1312-1320. 
27 Nakamura M, Nishikawa J, Hamabe K, Nishimura J, Satake M, Goto A, et al: Risk factors for delayed bleeding from endoscopic submucosal dissection of gastric neoplasms. Scand J Gastroenterol 2012;47: 1108-1114.

28 Toyokawa T, Inaba T, Omote S, Okamoto A, Miyasaka R, Watanabe K, et al: Risk factors for perforation and delayed bleeding associated with endoscopic submucosal dissection for early gastric neoplasms: analysis of 1123 lesions. J Gastroenterol Hepatol 2012;27:907912.

29 Jang JS, Choi SR, Graham DY, Kwon HC, Kim MC, Jeong JS, et al: Risk factors for immediate and delayed bleeding associated with endoscopic submucosal dissection of gastric neoplastic lesions. Scand J Gastroenterol 2009;44:1370-1376.

30 Hotta K, Oyama T, Akamatsu T, Tomori A, Hasebe O, Nakamura N, et al: A comparison of outcomes of endoscopic submucosal dissection (ESD) for early gastric neoplasms between high-volume and low-volume centers: multi-center retrospective questionnaire study conducted by the Nagano ESD Study Group. Intern Med 2010;49:253259.

31 Takizawa K, Oda I, Gotoda T, Yokoi C, Matsuda T, Saito Y, et al: Routine coagulation of visible vessels may prevent delayed bleeding after endoscopic submucosal dissection-an analysis of risk factors. Endoscopy $2008 ; 40$ : 179-183.
32 Oda I, Saito D, Tada M, Iishi H, Tanabe S, Oyama T, et al: A multicenter retrospective study of endoscopic resection for early gastric cancer. Gastric Cancer 2006;9:262-270.

33 Chung IK, Lee JH, Lee SH, Kim SJ, Cho JY, Cho WY, et al: Therapeutic outcomes in 1000 cases of endoscopic submucosal dissection for early gastric neoplasms: Korean ESD Study Group multicenter study. Gastrointest Endosc 2009;69:1228-1235.

34 Mochizuki S, Uedo N, Oda I, Kaneko K, Yamamoto Y, Yamashina T, et al: Scheduled second-look endoscopy is not recommended after endoscopic submucosal dissection for gastric neoplasms (the SAFE trial): a multicentre prospective randomised controlled non-inferiority trial. GUT 2015;64:397-405. 